\title{
Quantifying the error of the core-valence separation approximation
}

\author{
Michael F. Herbst ${ }^{1,}$ a) and Thomas Fransson 2 ,b) \\ 1) CERMICS, École des Ponts ParisTech, $6 \& 8$ avenue Blaise Pascal, 77455 Marne-la-Vallée, France; Inria Paris, \\ 75589 Paris Cedex 12, France; Sorbonne Universitée, Institut des sciences du calcul et des données, ISCD, \\ 75005 Paris, France \\ ${ }^{2)}$ Interdisciplinary Center for Scientific Computing, Heidelberg University, 69120 Heidelberg, Germany; Fysikum, \\ Stockholm University, Albanova, 10691 Stockholm, Sweden
}

For the calculation of core-excited states probed through X-ray absorption spectroscopy, the core-valence separation (CVS) scheme has become a vital tool. This approach allows to target such states with high specificity, albeit introducing an error. We report the implementation of a post-processing step for CVS excitations obtained within the algebraic-diagrammatic construction scheme for the polarisation propagator (ADC), which removes this error. Based on this we provide a detailed analysis of the CVS scheme, identifying its accuracy to be dominated by an error balance between two neglected couplings, one between core and valence single excitations and one between single and double core excitations. The selection of the basis set is shown to be vital for a proper description of both couplings, with tight polarising functions being necessary for a good balance of errors. The CVS error is confirmed to be stable across multiple systems, with an element-specific spread for $K$-edge spectrum calculations of only about $\pm 0.02 \mathrm{eV}$. A systematic lowering of the CVS error by $0.02-0.03 \mathrm{eV}$ is noted when considering excitations to extremely diffuse states, emulating ionisation.

\section{INTRODUCTION}

For the study of the electronic and atomic structure of materials, spectroscopy methods in the X-ray regime have recently seen key advances ${ }^{1}[\underline{4}$ By probing the transition of core electrons to (bound) excited states techniques such as X-ray absorption spectroscopy (XAS) provide information on the nature of unoccupied states surrounding specific elements. ${ }^{2|3| 5|6|}$ The use of core-orbital resonance energies, which are highly characteristic for the elements, thus provides a local and sensitive tool for investigating electronic structure.

In order to accurately model the core-excitation processes including electron relaxation effects is vital. This requires a theoretical method capable of capturing two aspects: Firstly, a reduced screening of the probed nuclei following the removal of a core electron, leading to a net attraction of the electron density to the core. Secondly, a smaller repulsive polarisation effect in the valence region due to interaction with the excited electron. These counteracting effects have to be properly accounted for in a theoretical framework, either by explicitly optimising the excited state or by introducing (at least) doubly excited configurations. On top of these modelling difficulties a practical challenge is that core-excited eigenstates are embedded in a continuum of valence-excited states, which prohibits a bottom-up solution of all sought core-excited for all but the smallest of systems $\frac{5}{5}$ Despite these challenges many theoretical methods for simulating core-excited spectra have been developed and made available. $517-21$

One approach which has proved to be successful is the core-valence-separation (CVS) scheme, $\frac{17 / 18}{1}$ in which the

\footnotetext{
a) Electronic mail: michael.herbst@inria.fr

b) Electronic mail: thomas.fransson@iwr.uni-heidelberg.de
}

weak electrostatic coupling between core- and valenceorbitals allows certain electron-repulsion integrals (ERI) to be neglected. Amongst a reduction in the involved matrix dimensions and thus a decrease in computational cost, this approximation decouples the valence continuum from core-excited states allowing to target specifically only the latter kind of excitations. The CVS approximation has been successfully implemented using a variety of wave function and density based methods, $\frac{5}{5}$ including coupled cluster theory, ${ }^{15 / 16}$ density cumulant theory, ${ }^{[12}$ multireference theory, ${ }^{11}$ time-dependent density functional theory ${ }^{7}$ and the algebraic-diagrammatic construction scheme.17 20122123 Note that the application of a core-valence separation scheme is not unique, $\frac{16}{1}$ and thus may vary between implementations.

Due to the neglected ERIs or - as an effect - the neglected coupling between core and valence excitation classes, the CVS approximation introduces an error to the obtained core excitation energies. Using a range of approaches such as perturbation theory ${ }^{18}$ damped response theory, ${ }^{2425}$ real-time propagation approaches, ${ }^{26}$ the Lanczos approach, $\frac{13}{2}$ or by considering the full-space problem, 12 the CVS error has been reported to range from close to zero to about $1 \mathrm{eV}$, depending on the employed wave function method, system, CVS implementation and basis set. It should be noted, however, that these error values have been found by considering systems of limited size and comparatively small basis sets. Furthermore, applying these methods to larger systems and basis sets may result in the complication that the core-excited states are no longer well-separated from the valence continuum, such that spurious valence-excited states need to be carefully identified and removed from the region of interest. ${ }^{27 / 28}$ This makes an estimation of the CVS error for basis sets including polarisation or augmentation rather involved, and such an assessment of the CVS approximation for larger basis sets of such types is missing 
to date.

To overcome this limitation this work presents a postprocessing step based on Rayleigh-Quotient iteration, 29 which amounts to undo the CVS approximation and refine obtained CVS eigenvectors back to the respective full excitation vectors. For our study of the CVS error we will apply this CVS relaxation in the context of the algebraic-diagrammatic construction scheme for the polarisation propagator (ADC) using the intermediate state representation (ISR) ${ }^{30 \mid 31}$. This family of methods has been demonstrated to give very good agreement with experiment $\frac{19|20| 22 \mid 32}{2}$ and it allows a large number of properties in the core region to be tackled! $!^{[9|20| 22|25| 33 \mid 35]}$ Due to the similarities between ADC and other post-HF methods such as coupled-cluster theory, we believe both our CVS relaxation methodology as well as our results for the CVS error to be applicable beyond the scope of ADC.

The outline of this paper is as follows: Section II summarises the core-valence separation in the context of ADC and introduces our methodology for CVS relaxation. Section III lists the computational methodology for our study of the CVS error on representative compounds with elements of the second and third period employing primarily double and triple-zeta basis sets including (core) polarisation and augmentation. The obtained results are summarised and discussed in Section IV.

\section{CVS RELAXATION IN THE ADC CONTEXT}

The key equation to be solved for the ADC scheme is the Hermitian eigenvalue problem 36

$$
\mathbf{M} \vec{X}_{i}=\Omega_{i} \vec{X}_{i}, \quad\left\langle\vec{X}_{i} \mid \vec{X}_{j}\right\rangle=\delta_{i j},
$$

where one obtains the excitation energies as eigenvalues $\Omega_{i}$ and the excitation vectors $\vec{X}_{i}$ as eigenvectors of the socalled ADC matrix $\mathbf{M}$. The structure and shape of the ADC matrix $\mathbf{M}$, thus the difficulty of (1), depends on the $\mathrm{ADC}(n)$ method under consideration. Commonly its size prevents a full diagonalisation, $\frac{36}{3}$ such that iterative methods are employed. Describing valence excitations at $\operatorname{ADC}(n)$ level is usually straightforward, since their corresponding excitation energies are located at the bottom end of the spectrum of $\mathbf{M}$ making them easily accessible. In contrast, core-excited states have higher energies and are interior eigenvalues of $\mathbf{M}$, such that computing them by iterative diagonalisation of (1) is challenging.

This picture changes if one considers the CVS approximation. Following Barth and Schirmer ${ }^{18}$ the essence of the CVS scheme is to neglect the interaction of core and valence orbitals via the Coulomb kernel by setting a number of electron-repulsion integrals explicitly to zero. As a result one part of the ADC matrix, which describes core-excitation processes may be completely decoupled from the remainder ${ }^{[37}$ This is summarised in Figure 1 for the $\mathrm{ADC}(3)$ matrix of water. Denoting by "o" a valence-occupied orbital, by "c" a core-occupied orbital and by "v" a virtual orbital, only the parts of the ADC matrix describing the interaction between cv singly and cvov doubly excited configurations remain in the CVS$\operatorname{ADC}(3)$ matrix $\mathbf{m}$. Notice, that other CVS schemes differ at this point and might, for example, also include the $\mathrm{cvcv}$ doubles excitations in the reduced matrix m. Excitation energies and excitation vectors of core-excited states may now be obtained by solving the smaller eigenproblem

$$
\mathbf{m} \vec{x}_{i}=\omega_{i} \vec{x}_{i}, \quad\left\langle\vec{x}_{i} \mid \vec{x}_{j}\right\rangle=\delta_{i j},
$$

where lower-case symbols are used for the equivalent quantities of (1). Apart from the reduced size an advantage of the CVS approximation is that the eigenpairs of interest are again located at the lower end of the spectrum of $\mathbf{m}$, such that the same iterative methods to compute valence-excitations in $\mathbf{M}$ can now be used to compute core-excitations in $\mathbf{m}$.

A consequence of the CVS scheme being an approximation is that the CVS excitation vector $\vec{x}_{i}$ is not an eigenvector of the full ADC matrix $\mathbf{M}$. The observed validity of the approximation $12|15| 18 \mid 25$ suggests, however, that it should already be very close. A good starting point for a refinement procedure resulting in the full excitation vector $\vec{X}_{i}$ and corresponding full excitation energy $\Omega_{i}$, is thus to start from an already obtained CVS vector $\vec{x}_{i}$. This step, the CVS relaxation, thus effectively undoes the CVS approximation contained in $\vec{x}_{i}$.

For this purpose we employ a simple scheme based on Rayleigh-Quotient iteration.29 applied to one CVS eigenvector at a time. Starting from the CVS quantities $\omega^{(0)}=\omega$ and $\vec{x}^{(0)}=\vec{x}$, we iterate as follows:

1. Solve the equation

$$
\left[\mathbf{M}-\left(\omega_{i}^{(n-1)}+\varepsilon\right) \mathbf{I}\right] \alpha_{i}^{(n)} \vec{x}_{i}^{(n)}=\vec{x}_{i}^{(n-1)}
$$

for $\vec{x}_{i}^{(n)}$, using a conjugate-gradient algorithm preconditioned with the diagonal of the shifted ADC matrix. In this $\alpha_{i}^{(n)}$ is chosen to keep $\vec{x}_{i}^{(n)}$ normalised and $\varepsilon$ is a small positive constant to improve conditioning.

2. Compute the updated eigenvalue estimate $\omega_{i}^{(n)}=\vec{x}_{i}^{(n) T} \mathbf{M} \vec{x}_{i}^{(n)}$. Converge if residual norm $\left\|\mathbf{M} \vec{x}_{i}^{(n)}-\omega_{i}^{(n)} \vec{x}_{i}^{(n)}\right\|$ is below a threshold, else increment $n$ and return to 1 .

During the iterations we monitor the overlap $\left\langle\vec{x}^{(n)} \mid \vec{x}\right\rangle$ to ensure we stay close to the state of interest. If this value gets too small we restart the algorithm with based on the iterate of largest overlap and a refined guess for $\omega^{(0)}$.

The discussed relaxation scheme is general and could be applied to any Hermitian eigenvalue problem, including alternative formulations of the CVS approximation or the eigenproblem arising in TDDFT. Moreover, CVS relaxation in the context of coupled-cluster could be approached similarly if equivalent algorithms suitable for 

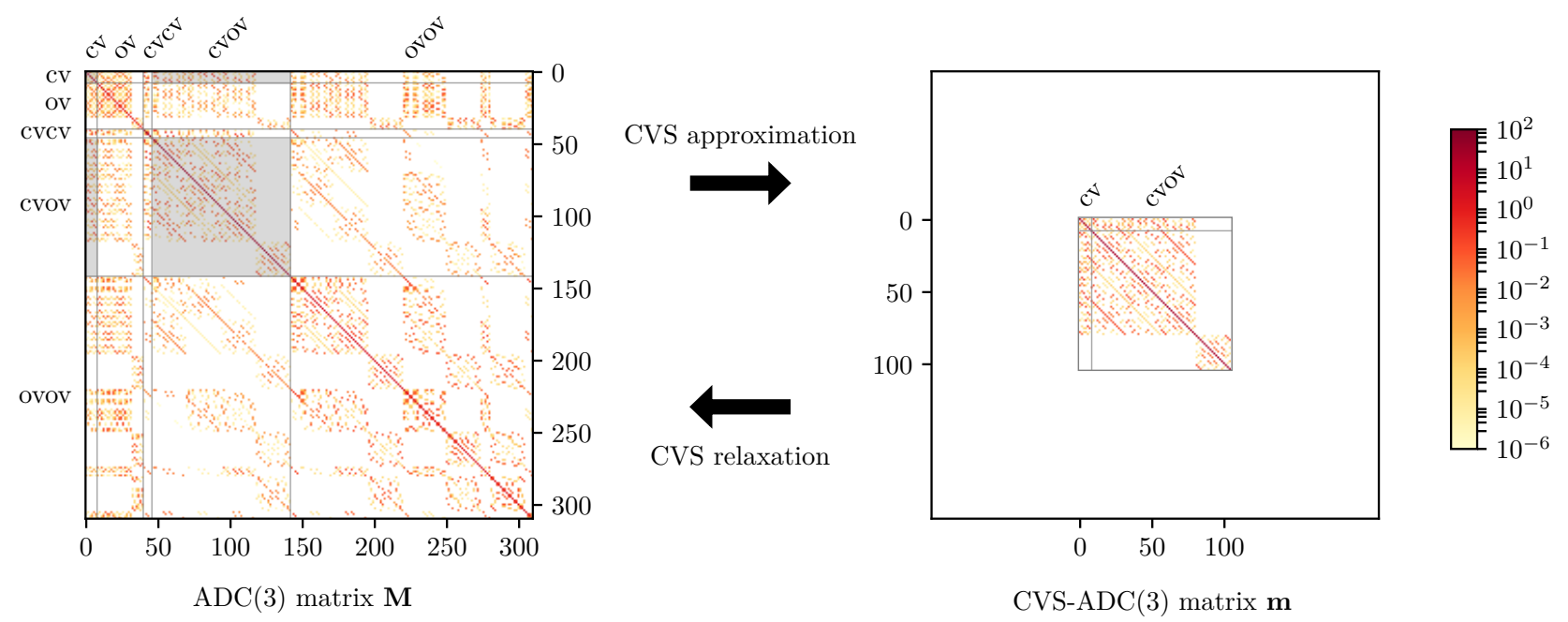

FIG. 1. Full ADC(3) matrix and CVS-ADC(3) matrix of water using a STO-3G $\sqrt[38]{38}$ basis obtained using adcc. ${ }^{39}$ Grey lines separate blocks of different single- and double-excitation classes with "c" denoting excitation from a core orbital, "o" from a valence orbital, and "v" excitation into a virtual orbital. Blocks of the full matrix $\mathbf{M}$, which are present in the CVS matrix $\mathbf{m}$ as well are marked by a grey background.

non-Hermitian matrices are employed, such as the generalised minimal residual method ${ }^{29}$ for solving (3). We remark that we selected this scheme mainly due to its simplicity and generality. If a black-box application of the CVS relaxation in production calculations was desired we would expect further improvements with respect to reliability and performance to be necessary.

\section{COMPUTATIONAL DETAILS}

Molecular structures have been optimised at the $\mathrm{MP}^{401} / \mathrm{cc}^{4 \mathrm{pVT}} \mathrm{Z}^{41}$ level of theory, using the Q-Chem ${ }^{42}$ program. Calculations of core-excited states have been performed in the adcc python package ${ }^{39 \mid 43}$ using HartreeFock (HF) references obtained in pyscf. ${ }^{[4]}$ For comparing CVS errors, excitation vectors are first obtained at either CVS-ADC(1), CVS-ADC(2), CVS-ADC(2)-x or CVS-ADC(3) level of theory using adcc and afterwards relaxed to the full ADC level of theory using a Python implementation of the CVS relaxation of Section II. Absorption cross-sections were computed using the intermediate state representation (ISR) ${ }^{30}$. This implementation is available on github ${ }^{45}$ and will be integrated into adcc in the future. For our study we employed the $6-311++\mathrm{G}^{* * 46}$ and the Dunning family of basis sets, ${ }^{41}$ including core-polarising ${ }^{47}$ and diffuse ${ }^{48}$ functions. Where values are compared to experiment, relativistic effects were estimated by calculating the $1 \mathrm{~s} \mathrm{MO}$ energies at a HF/cc-pCVTZ level of theory in both a non-relativistic framework and a scalar relativistic framework using the second-order Douglas-Kroll-Hess Hamiltonian 4951 These calculations were performed in the
Dalton quantum chemical program. 52153

\section{RESULTS AND DISCUSSION}

\section{A. Components of the core-valence separation error}

To leading order, core-excitations are described by the $\mathrm{ADC}$ matrix elements in the cv-cv block of the ADC matrix. Within the core-valence separation scheme discussed in Section II only the interaction of the cv-part of the excitation vector to the cvov doubly excited configurations is taken into account. Inside the singles block of the ADC matrix it is thus the coupling to the ov configurations and inside the doubles block to both the cvcv and the ovov configurations, which are neglected and make up the CVS error. The cv-ovov coupling can be argued to be extremely small due to both the energetic as well as the spatial separation of the core and valence orbitals ${ }^{17}$ and will henceforth be ignored in our discussion. Similarly all higher-order couplings involving doubles excitations from different blocks are small and will be ignored. To leading order we are thus left with the effects from neglecting the cv-ov and the cv-crcv coupling within the CVS approximation. Starting from the CVS result as a reference, a perturbation theory argument (see Appendix) allows to draw two conclusions: (1) The cv-ov coupling pushes the energy of the core-excitations $u p$, since the ov singles excitations are energetically below the $\mathrm{cv}$, and (2) the cv-crcv coupling pushes the energy down, since the crcv doubles have an excitation energy above the cv singles excitations. The neglected couplings therefore have opposing effects with respect to the total CVS error. We 
note that the present discussion is focused on the $K$-edge where the cv block only consists of $1 s$ core excitations. For the $L$-edge additional aspects, such as the coupling between inner core and outer core regions inside the cv block, can be expected to influence the error balance as well.

For obtaining separate estimates for both sources of the CVS error we perform two different kinds of CVS relaxations. First a conventional CVS-ADC calculation followed by a CVS relaxation, yielding the total CVS error. Second a calculation, where only the singles block of a particular ADC level of theory is used in both the CVS-ADC calculation as well as the relaxation. We will refer to the error observed in this case as the singles-block $C V S$ error. By construction the results of the singles-only CVS calculation differ from their relaxed counterparts only by neglecting the cv-ov coupling. To leading order the singles-block error is therefore equal to the part of the total CVS error originating from the neglected cv-ov coupling. This implies that the difference between total and singles-block CVS error provides a leading-order estimate to the error from neglecting the cv-cvcv coupling in the CVS treatment.

To illustrate the magnitude of these effects, Figure 2 reports the CVS errors of the 10-electron series, using an exhaustive aug-cc-pCVTZ basis on the probed atom and cc-pVTZ on the hydrogens. Errors have been obtained for one state for each molecule, save $\mathrm{NH}_{3}$ and $\mathrm{H}_{2} \mathrm{O}$, for which two states were included. The top panel provides an overview of the total CVS error for $\mathrm{ADC}(2)$, the singles-block CVS error as well as the difference between these. As expected from our discussion, the values of the singles-block CVS error are negative, meaning that the introduction of the cV-Ov coupling during relaxation raises the energy of the core excitation. In turn, the difference as a measure for the cv-cvcv coupling has the opposite sign. We note that in absolute values both errors are of similar size, amounting to approximately $1.2 \mathrm{eV}$. Tests using a core-polarised quadruple-zeta basis set also yields a small total CVS error. As will be detailed in Section IV C this is not an artefact, much rather it suggests that a balanced description of both terms is key to obtain a small CVS error. In the bottom panel of Figure 2 the singles-block errors for $\mathrm{ADC}(1), \mathrm{ADC}(2)$ and $\mathrm{ADC}(3)$ are reported. For $\mathrm{ADC}(1)$ the error is small, while $\mathrm{ADC}(2)$ and $\mathrm{ADC}(3)$ have single-block errors of similar size, being larger for $\operatorname{ADC}(2)$. This is not surprising, since the off-diagonal elements for the $\mathrm{ADC}(1)$ matrix are only made up of the Coulomb and exchange integrals, which are especially small for the cv-ov coupling block.

\section{B. CVS error behaviour in the $\operatorname{ADC}(n)$ hierarchy}

Figure 3 summarises the CVS errors (full, singlesblock, and difference) calculated using the $6-311++\mathrm{G}^{* *}$ basis set. We selected this basis set because it has
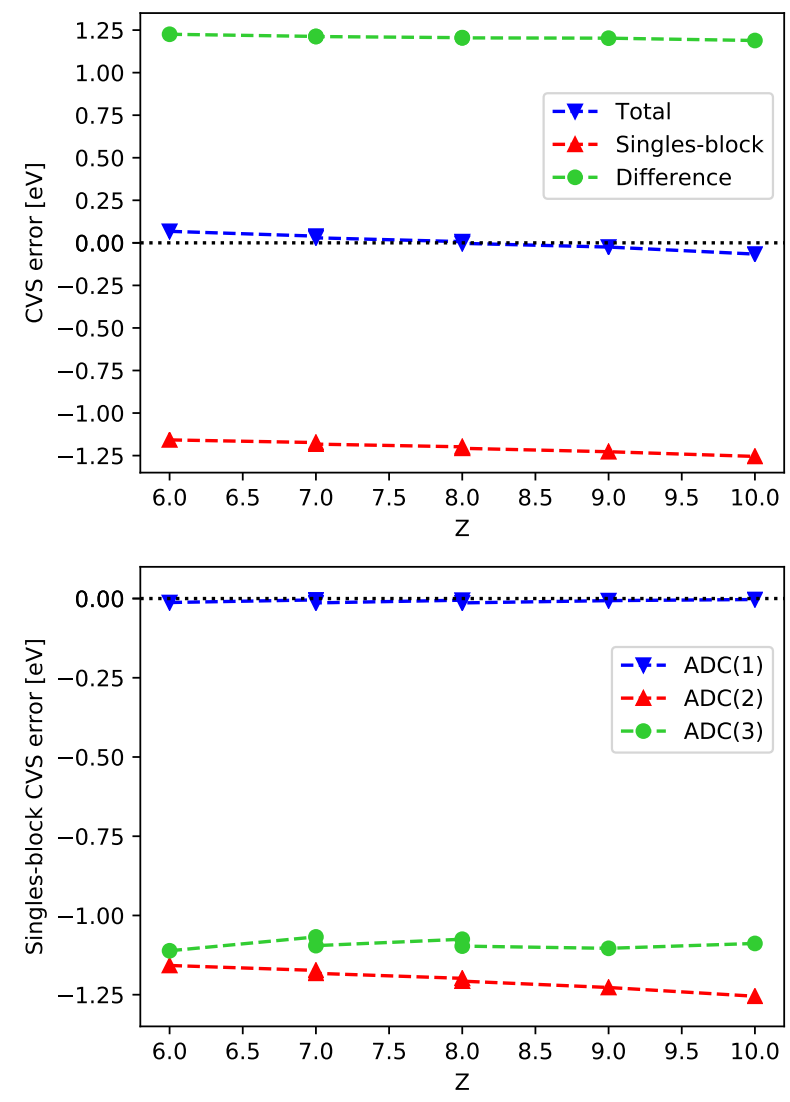

FIG. 2. The CVS error of the 10-electron series, using an aug-cc-pCVTZ basis set on C, N, O, F, and Ne, and cc-pVTZ on H. Top: CVS error broken down to full error, singles-block error, and error difference, obtained using $\mathrm{ADC}(2)$. Bottom: Singles-block CVS error of the ADC hierarchy.

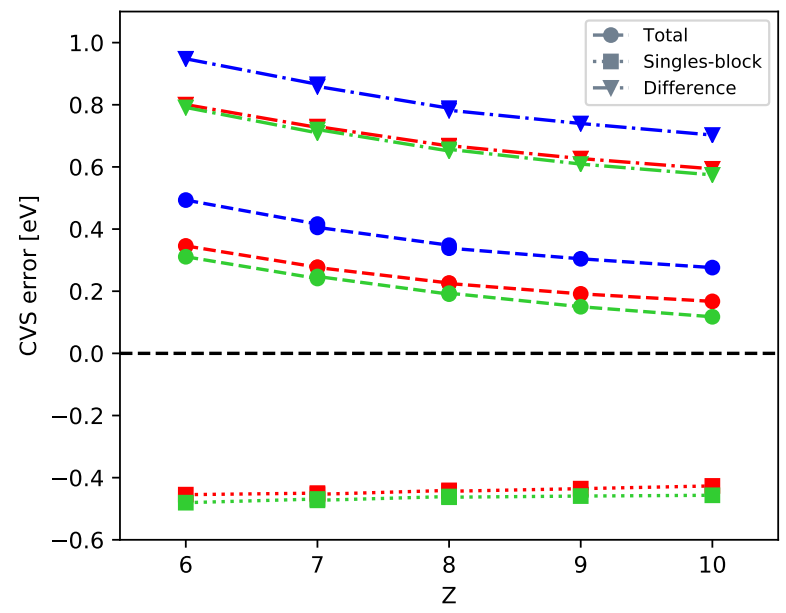

FIG. 3. Components of the CVS error of $\mathrm{ADC}(2)$ (blue), $\mathrm{ADC}(2)-\mathrm{x}$ (red), and $\mathrm{ADC}(3)$ (green), as a function of atomic number Z. Results obtained for the 10-electron series, using the $6-311++\mathrm{G}^{* *}$ basis set. 
been shown to give good agreement with experiment at a relatively low computational cost, making it important for production calculations 16120132154 It should be noted that all observed trends could be reproduced with a cc-pCVTZ basis.

With respect to the CVS error we observe the trend $\operatorname{ADC}(2)>\operatorname{ADC}(2)-\mathrm{x}>\mathrm{ADC}(3)$ for the total error as well as all components. Most notably, the increased order in perturbation theory in the doubles block going from $\mathrm{ADC}(2)$ to $\mathrm{ADC}(2)$-x causes a clear drop of total CVS error towards the value observed with $\operatorname{ADC}(3)$. In this basis the singles-block error turned out to be almost agnostic to the ADC variant (the singles-block CVS error is identical for $\operatorname{ADC}(2)$ and $\operatorname{ADC}(2)-x$, by construction), such that the difference in errors shows a similar behaviour as the total error. Recalling the error difference to be a measure for the cv-cvev coupling we attribute the observed trend to a compensation of the neglected cv-cvcv coupling by the higher-order treatment of cvov excitation in CVS-ADC(2)-x and beyond. The error in the description of core-relaxation effects, to which the cv-cvev couplings contribute, can thus be expected to be smaller at the CVS-ADC(2)-x and CVS-ADC(3) level than at CVS-ADC(2). In terms of absorption crosssections, the CVS results typically underestimate the intensities obtained in a full treatment. For the systems presented in Figure 3 for example, we report discrepancies from $-6 \%$ to $+2 \%$, with an overestimation observed only for neon. The trend of a lowering of intensities (with the exception of neon) is observed for all our calculations, with discrepancies typically being lower for larger basis sets and for third row elements. This suggests that the behaviour is general, and our discussion of the CVS error thus focuses on excitation energies.

For the singles-block CVS error of $\operatorname{ADC}(2)$ and $\operatorname{ADC}(3)$, we performed a more exhaustive study using the cc-pCV $n \mathrm{Z}(n=\mathrm{D}, \mathrm{T}, \mathrm{Q})$ series of core-polarised Dunning basis sets, illustrated in Figure 4 Complete basis set extrapolations are included for the systems, where our CVS relaxation procedure managed to converge the cc-pCVQZ states. In general, the size of the singles-block CVS error increases with the size of the basis. Over the range of considered elements it is relatively constant if a double-zeta basis is used. For larger bases the discrepancy increases with $Z$ for $\operatorname{ADC}(2)$, while trends are less clear for $\mathrm{ADC}(3)$. Between $\mathrm{ADC}(2)$ and $\mathrm{ADC}(3)$ the magnitude of the errors are similar for cc-pCVDZ, with $\mathrm{ADC}(3)$ resulting in slightly larger values. In the larger basis sets, however, the ordering and relative sizes are markedly different - here the $\mathrm{ADC}(3)$ error is significantly smaller, especially using cc-pCVQZ. The error component due to the neglected cv-ov coupling is thus smaller in CVS-ADC(3) compared to lower-order CVS-ADC variants. Similar to what was observed for the cv-cvev error in contrast to the order of perturbation theory in the doubles block, this suggests that a treatment of the singles-block at higher order is favourable to recover some effects of the neglected cv-ov interaction, In

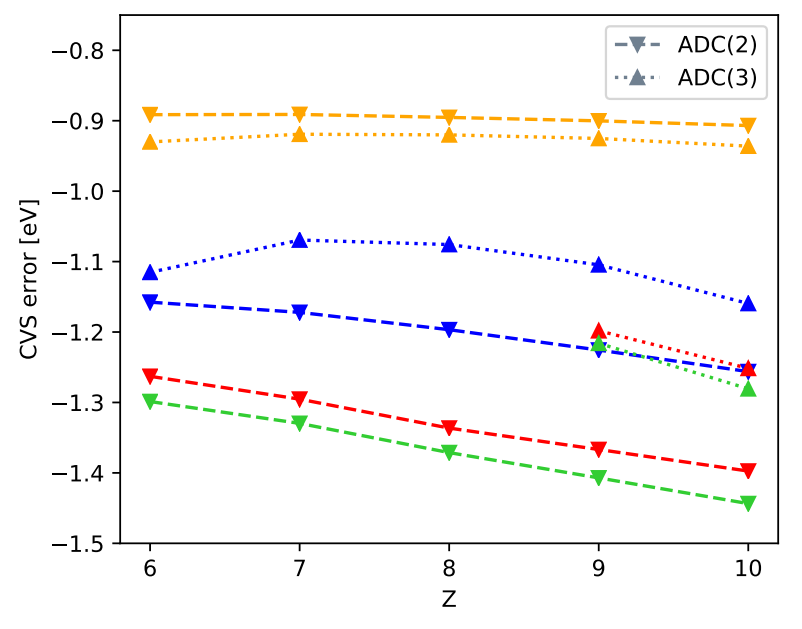

FIG. 4. Singles-block CVS error of $\mathrm{ADC}(2)$ and $\mathrm{ADC}(3)$, as obtained using cc-pCVDZ (orange), cc-pCVTZ (blue), and ccpCVQZ (red) basis sets, as well as for a CBS estimate (green). Results obtained for the 10-electron series.

our subsequent analysis we will focus on the CVS error in $\operatorname{ADC}(2)$, where the results presented here have identified both CVS error components to be largest.

\section{Basis set effects}

Suitable basis sets for core-excitations and coreionisations are well-investigated, with some tendency to favour IGLO,,$[556$ basis sets formed by inclusion of functions from the next element, $\frac{\sqrt{57}}{,}$ or amending standard basis sets by core-polarising functions. 16156158 For our investigation of the CVS error we have focused on 6-311++ $\mathrm{G}^{* *}$ and basis sets from the Dunning family. The former is commonly used for X-ray spectroscopies, and the latter is known to converge rigorously for correlated methods and offer a standardised way to include core-polarising and diffuse functions $[59$ Motivated by previous results with modified $6-311++\mathrm{G}^{* *}$ basis sets yielding good agreement with experiment, $\frac{35|56| 60 \mid 61}{16}$ we also included three modifications on $6-311++\mathrm{G}^{* *}$, namely (1) $\mathrm{u} 6-311++\mathrm{G}^{* *}$, where the $1 s$ basis functions have been decontracted, (2) p6-311++ $\mathrm{G}^{* *}$, where on top of $6-311++\mathrm{G}^{* *}$ we added another six $p$-functions with exponents taken from the $1 s$ functions, and (3) up6-311 $++\mathrm{G}^{* *}$, formed by both decontraction and addition of six $p$-functions. ${ }^{62}$ These allow to probe the flexibility required in the core region for obtaining a low CVS error. Our results for the 10-electron (18-electron) series of the second-row (third-row) elements at the $\operatorname{ADC}(2)$ level are shown in Figure 5. The trends in the total CVS error (top panel) can be best explained by considering them as the sum of the trends in the singles-block CVS error (middle panel) and the difference error (bottom 


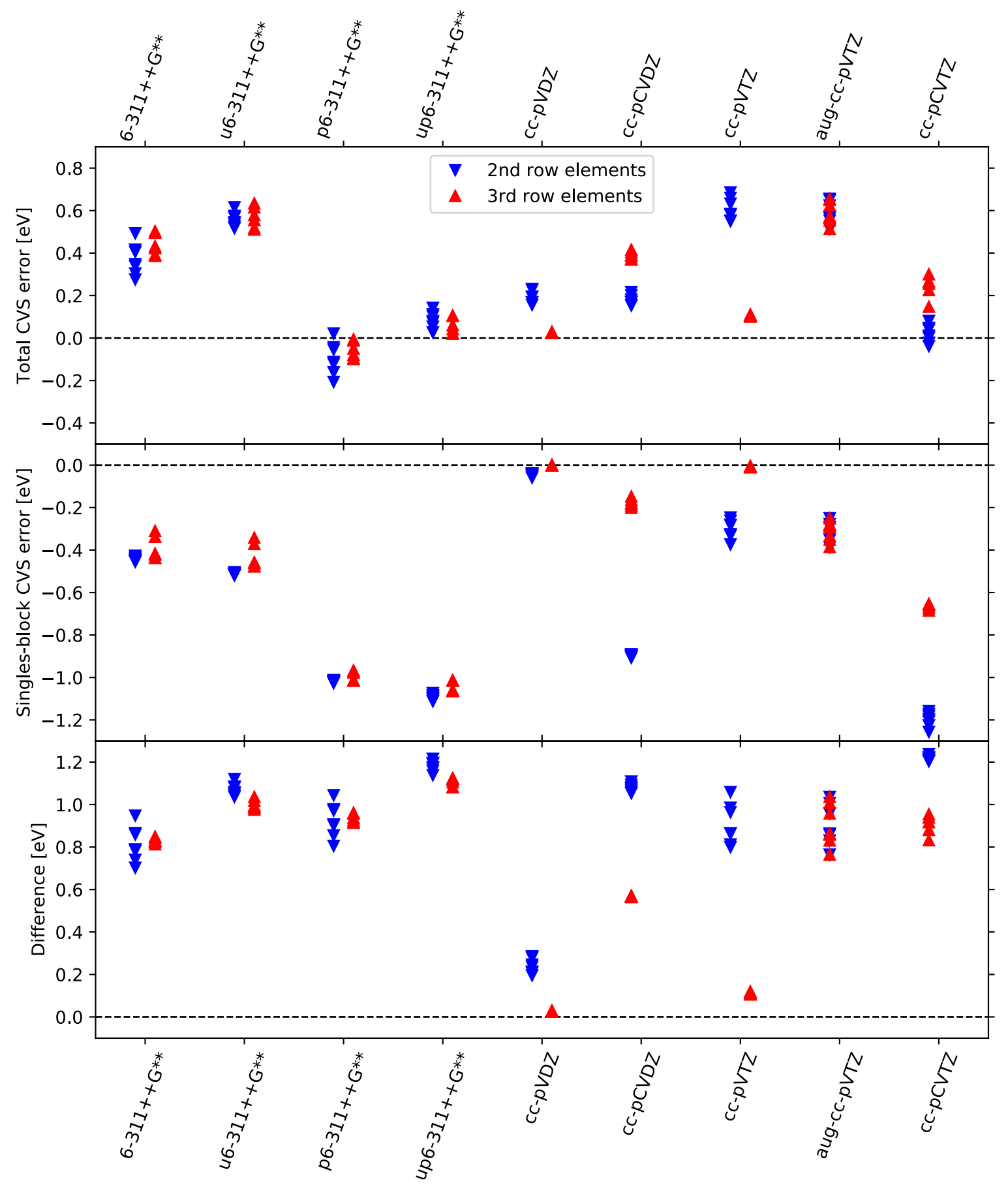

FIG. 5. The components of the $\mathrm{ADC}(2) \mathrm{CVS}$ error, as obtained for the 10- and 18-electron series using a number of different basis sets. Note that all panels are to scale.

panel). Since these latter measures are directly related to the cv-Ov and cv-crcv couplings, it is in fact the balance between the ability of a basis set to describe the $\mathrm{cr}$ and ov singles and cvcv doubles excitations, which plays the key role. This in turn depends on the flexibility of the basis set to describe both core and valence region in a well-adjusted manner, such that relaxation and correla- tion effects in the core as well as the valence region can be described.

Since most basis sets focus on a description of the chemically most interesting valence region, for small basis sets cv- and ov-excitations are not described equally well. As basis sets get larger or as extra functions in the core region are added (e.g. by core polarisation), the 
description of the core orbitals catches up, leading to a drop in cv-excitation energies relative to the ov-excitation energies. As a result the gap between them decreases and the cv-ov coupling and the singles-block CVS error increases. In our results this can be observed in the change in singles-block CVS error between $6-311++\mathrm{G}^{* *}$ and $\mathrm{p} 6-6-311++\mathrm{G}^{* *}$, where the only change to the basis is the addition of polarising $p$-functions in the core region. Other examples are the change between cc-pVDZ and cc-pCVDZ, as well as cc-pVTZ and cc-pCVTZ.

Along similar lines one would expect an improved description of the core region to increase the cv-cvcv coupling, since the cvcv doubles excitations are twice as effected by the drop in core orbital energies as the cv singles excitation. In this aspect the trends in our results for the difference between full and single-block CVS error are not so clear. Still, the largest error differences in our study are observed for cc-pCVTZ and up6-311++G**, i.e. the basis sets with largest flexibility in the core region we consider, which suggests that basis sets providing a fuller description of the core region will indeed have a larger error difference. Additionally, most other basis sets also yield difference values in reasonable agreement with cc-pCVTZ and up6-311++ $\mathrm{G}^{* *}$, the main outliers being cc-pVDZ for all elements, and cc-pCVDZ and cc-pVTZ for the third row elements. Surprising is the significant shift in CVS error difference when adding diffuse functions to cc-pVTZ for the third row elements, with average error changing from about $0.1 \mathrm{eV}$ to $1 \mathrm{eV}$. One would not expect the cv-cvcv coupling to be particularly affected by the addition of diffuse functions.

With respect to the full CVS error, both neglected couplings contribute with opposite sign, thus giving rise to an even more unsystematic behaviour. The smallest observed errors result if a basis describes cv-Ov and $\mathrm{cv}-\mathrm{cvcv}$ coupling either equally good (e.g. cc-pCVTZ for the second row) or equally bad (e.g. cc-pVDZ for the third row). It is thus the basis sets with large, but similar, magnitudes in the singles-block error and the error difference, which offer a description of the core region, where corerelaxation is accounted for as best as possible, but still a small total CVS error results in a CVS scheme. According to this metric the best basis for the second-row elements in our study is cc-pCVTZ, whereas for the thirdrow elements it is up6-311++ $\mathrm{G}^{* *}$. For cc-pCVTZ the total CVS errors values are distributed as $0.02 \pm 0.04 \mathrm{eV}$ for the second row and $0.24 \pm 0.06 \mathrm{eV}$ for the third row, and for up6-311++ $\mathrm{G}^{* *} 0.08 \pm 0.04 \mathrm{eV}$ and $0.06 \pm 0.04 \mathrm{eV}$, respectively. If smaller basis sets are desired, cc-pCVDZ is reasonable for the second row, but again p6-311++ $\mathrm{G}^{* *}$ is the better choice for the third row. We note that the poor performance for cc-pCVDZ and cc-pCVTZ for the third-row elements is not surprising, since the corepolarising functions are the result from minimising the amount of core-core and core-valence correlation combined $\sqrt[59163]{ }$ thus putting more emphasis on the outer core region. Our study, however, probes the inner core region with $1 s$ core excitations, making the standard core- polarised Dunning basis sets less applicable for the thirdrow elements.

Summarising the trends obtained over the basis sets in Figure 5, we find that properly describing the cv-ov coupling is more challenging than the cv-cvev. Additional $p$-functions or the core-polarised Dunning basis sets are clearly required to describe the core and valence region on a similar level. Considering specifically the $6-311++\mathrm{G}^{* *}$ basis set and modifications thereof, we note that the addition of tight $s$-functions only amounts to improve the description of the cv-cvcv coupling a little (raising the error difference), but it is the addition of the tight $p$ functions which drastically improves the ov-cv coupling and thus leads to a drop in total CVS error. We remark that these conclusions might be different for core spectroscopies probing $L$-levels, due to stronger coupling between $2 s$ and $2 p$, as well as the presence of a deeper lying $1 s$ - indeed, a recent publication on EA- and IP-ADC reported significantly larger CVS errors for ionisation of $2 s$ than of either $1 s$ or $2 p \underline{\underline{23}}$

From our results we expect CVS schemes which include the cr-cvcr coupling to lead to a negative CVS error, i.e. to an overestimation of the excitation energy for $1 s$-core excitations. This is especially the case for the larger and more adequate basis sets, where they will not benefit from the error cancellation obtained from neglecting both the cv-ov and the cv-crcv coupling. For smaller basis sets, the magnitudes of both cv-ov and cvcvcv couplings are generally smaller, such that we believe this aspect to be overlooked in previous analysis comparing CVS schemes.

\section{The CVS error of different compounds and states}

We now consider the element-specific spreads of the CVS error. In Figure 6 we summarise the performance of the CVS approximation for the carbon and oxygen edge of a number of different compounds. These results have been obtained using the cc-pCVDZ basis set with an additional diffuse $s$-function (exponent $10^{-11}$ ) for the probed element, and cc-pVDZ for all others. Intense transitions to bound states as well as ionisations are both included in our analysis with the latter being modelled by investigating transitions to the additional diffuse function ${ }^{[154}$ Trends observed in the cc-pCVDZ basis set, already confirmed to yield a reasonable description of both CVS error sources, could be reproduced with a up6-311++ $\mathrm{G}^{* *}$ basis set for selected cases. In agreement with our observation in Section IVB intensities for the presented compounds are underestimated by $1-4 \%$ when using the CVS approximation.

Over the range of considered compounds we note systematically lower full CVS errors for ionisation of about 0.02-0.03 eV. For all compounds the singles-block CVS error is of similar size, with bound excitations being about $0.01 \mathrm{eV}$ lower in energy. This minor difference in errors for both types of excitation processes is expected, since 

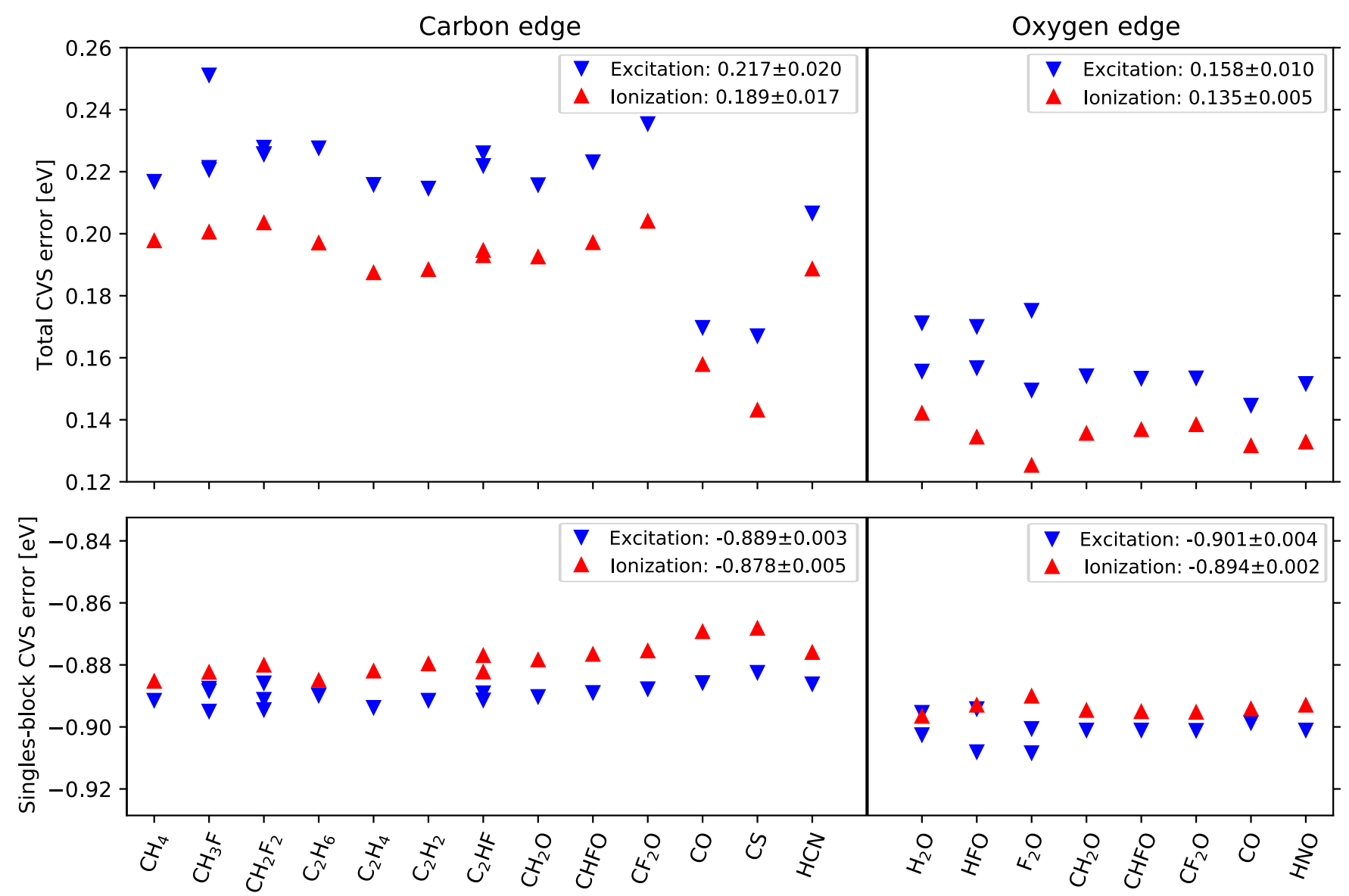

FIG. 6. The CVS error of $\mathrm{ADC}(2)$ for a number of compounds, as obtained using a cc-pCVDZ (with additional diffuse sfunction) basis set for the probed element, and cc-pVDZ for all other elements. Lower panel reports singles-block CVS error, using the same energy scale but different error boundaries. Upper right corner of each panel and edge reports the average error and standard deviation thereof.

both are dominated by the electronic structure of the core region and deviating aspect is the final state - once a delocalised $s$-function (ionisation) and once a localised valence orbitals. This also explains the smaller total CVS error observed for ionisations, since the cv-cvcv coupling is small between a delocalised cv-transition and cvcvdoubles excitations involving localised virtuals. Similarly we do not expect the CVS error for ionisation processes to change significantly if more sophisticated discretisation schemes for the continuum are employed. Adding additional diffuse functions, for example, resulted in a very quick convergence of the CVS error in our experiments.

Notice that our treatment of ionisations can be considered as a limiting case of transitions to diffuse Rydberg states. As such the $0.02-0.03 \mathrm{eV}$ difference in CVS error is the mean upper limit of the difference in CVS error between core-valence and core-Rydberg transitions. This is confirmed by rough tests on the core-Rydberg CVS error (not shown) yielding error values between that of excitations to valence states and ionisations. Overall this difference is negligible, being one order of magnitude smaller than the CVS error, which in turn is one order of magnitude smaller than the error with respect to experiment. We therefore do not expect this to have significant influence on obtaining a balanced description of the spectrum in CVS methods.

Considering the dependency of the CVS error on the compound and excitation, errors for the oxygen edge are similar across all molecules and transitions included here. These span excitation energies from $534.1 \mathrm{eV}$ (HNO) to $540.7 \mathrm{eV}$ (HFO). In simulated excitation spectra this is visible by a scalar shift of the complete spectrum when the CVS approximation is applied, see Figure 7. For the carbon edge, most errors are also found in a relatively narrow window, save $\mathrm{CO}$ and $\mathrm{CS}$ as the two outliers. For the latter two compounds the total CVS error is lower by about $0.05 \mathrm{eV}$, resulting in a larger CVS error spread for the carbon edge compared to the oxygen edge. Carbon transition energies span the range of $289.3 \mathrm{eV}$ (CS) to $297.8 \mathrm{eV}\left(\mathrm{CH}_{2} \mathrm{~F}_{2}\right)$ with the $\mathrm{CO}$ resonance occurring at $291.3 \mathrm{eV}$.

Using different basis sets, CO and CS remain outliers, similarly when changing for $\operatorname{ADC}(2)-x$ and $\operatorname{ADC}(3)$, al- 


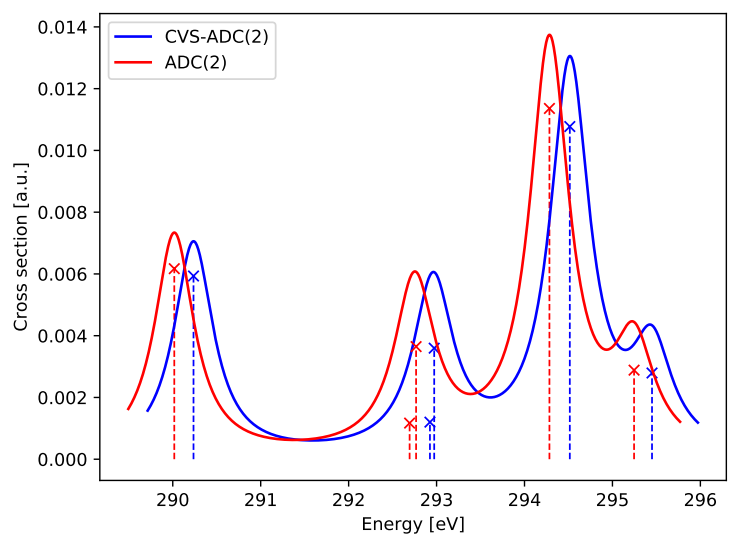

FIG. 7. Comparison of core-excitation spectrum of the carbon edge of 1,2-difluoroethane, as obtained at CVS-ADC(2) and $\mathrm{ADC}(2)$ levels using cc-pCVDZ for carbon and cc-pVDZ for all other elements. Peaks were broadened empirically using a Lorentzian line shape with width parameter 0.01 a.u.. Between both methods the spectrum is primarily just shifted by about $0.2 \mathrm{eV}$.

though the magnitude of the deviations diminishes. To a lesser extent $\mathrm{HCN}$ is also a weak outlier whereas $\mathrm{HNC}$ (not included in the figure) gives a total CVS error sitting between $\mathrm{CO}$ and $\mathrm{HCN}$. Additional calculations further show that the deviating CVS errors are not affected by restricting the CVS space to only the carbon $1 s$ or including also the nitrogen and sulphur $1 s$. We note that, e.g. fluorine substitution, which strongly shifts resonance energies, $\frac{58}{6}$ does not affect the CVS errors compared to related compounds. This indicates that distortion of the electronic structure near the core due to substitutions are not particularly influential on the CVS error, and we thus attribute the observed aberrations in $\mathrm{CO}$ and CS to the distorted electronic structure around specifically triple-bond systems. For practical purposes, these distorted situations are unlikely to be a concern, and the CVS error spread for carbon could thus be expected to be $\pm 0.01 \mathrm{eV}$, i.e. of similar magnitude as the oxygen edge. Furthermore, $\mathrm{C}_{2} \mathrm{H}_{2}, \mathrm{C}_{2} \mathrm{H}_{4}$ and $\mathrm{C}_{2} \mathrm{H}_{6}$ all have similar CVS errors as the other considered systems, despite the fact that the $1 s$ MOs are now delocalised over both carbon atoms. This is encouraging, as delocalised $1 s$ MOs can occur in larger systems. Notice that such delocalisations are more of a concern in simulations, as the real molecular system will always feature vibrations or other disorder, which typically breaks the degeneracies in the $1 s$.

\section{E. Comparison to previous analysis of the CVS error}

Previous studies have reported CVS errors of sizes varying from small negative values up to one $\mathrm{eV}$, in line with the order of magnitude we have obtained in the pre- vious Sections. Within the ADC framework the work of Barth and Schirmer ${ }^{[18}$ reports comparatively large errors $(0.5-1.0 \mathrm{eV})$ of $\mathrm{ADC}(2)$ using second-order perturbation theory. We account this to their work using a rather limited triple-zeta basis similar to cc-pVTZ, where we also obtain values of this order. Using damped response theory on $\operatorname{ADC}(2)$, Rehn, Dreuw, and Norman ${ }^{25}$ have estimated CVS errors for water of $0.19-0.35 \mathrm{eV}$ on $\mathrm{ADC}(2)$ using a $6-311++\mathrm{G}^{* *}$ basis, which agrees with our results.

In the context of coupled cluster theory, Coriani and co-workers have developed different CVS implementations. ${ }^{[5] 16 \mid 65}$ With linear-response coupled cluster and a scheme similar to ours, they report CVS errors of $0.04 \mathrm{eV}$ for neon, using an aug-cc-pCVTZ basis with additional diffuse functions ${ }^{15[65}$ Our results at the $\operatorname{ADC}(2)$ level is approximately $0.1 \mathrm{eV}$ lower, around $-0.06 \mathrm{eV}$. This is within the spread of a few tenths of an $\mathrm{eV}$ we observed in Section IVB comparing different ADC methods.

Peng, Copan, and Sokolov ${ }^{12}$ have recently developed two CVS schemes in the context of linear-response density cumulant theory, named CVS-ODC-12-a and CVSODC-12-b, and compared it to full ODC-12. CVS-ODC12 -a is similar to the CVS scheme we employ, neglecting both the cv-ov and the cv-crcv couplings, whereas CVS-ODC-12-b only neglects cv-ov. For variant -a one would therefore expect similar discrepancies as our total CVS errors, and for variant -b we instead expect values similar to our singles-block CVS error. Focusing on the first dipole-allowed feature of water using a 6-31G basis set, the quoted CVS error is $0.14 \mathrm{eV}$ for variant -a and $-0.01 \mathrm{eV}$ for variant $-\mathrm{b}$. Using $\mathrm{ADC}(2)$ we obtain a total CVS error of $0.19 \mathrm{eV}$ and a singles-block error of $-0.02 \mathrm{eV}$, whereas $\mathrm{ADC}(3)$ yields $0.07 \mathrm{eV}$ and $-0.04 \mathrm{eV}$, respectively, which is a reasonable agreement given the basis set.

\section{F. Comparison to experimental results}

A comparison of the performance of CVS-ADC $(2)$ and full $\mathrm{ADC}(2)$ with respect to experimental data is reported in Tables $\Pi$ and $\amalg$ for excitations to bound states and for ionisation potentials, respectively. For the excitations to bound states both CVS-ADC(2) and $\mathrm{ADC}(2)$ show a decreasing error along the elements of the second period. Neon deviates a little from this trend due to the inability of the employed basis to describe the $1 s \rightarrow 3 p$ transition properly. Since transition energies are overestimated for all systems and the CVS error is mostly positive, $\mathrm{ADC}(2)$ agrees a little better with experiment than CVS-ADC(2). For estimating the ionisation potential both ADC(2) models overestimate experiment for low $Z$ and underestimate it for high $Z$, again with CVS relaxation improving results a little. The larger discrepancy observed for ionisation is unsurprising, since it has been previously noted that fourth-order ADC methods are required for a good description of ionisation potentials. ${ }^{73}$ Clearly for both excitation and ionisation the CVS error is negligible compared to the total error with respect to 
TABLE I. Comparison of core-valence excitation energies obtained with ADC(2) and CVS-ADC(2) using a aug-cc-pCVTZ basis plus an additional diffuse $s$-function (exponent $10^{-11}$ ) on the probed element and cc-pVTZ on hydrogen. Energies are expressed in $\mathrm{eV},{ }^{\mathrm{a}} \Delta$ Expt denotes the error with respect to experimental values and $\Delta \mathrm{CVS}$ the total CVS error.

\begin{tabular}{|c|c|c|c|c|c|c|c|}
\hline \multirow[b]{2}{*}{ Molecule } & \multirow[b]{2}{*}{ Transition } & \multirow[b]{2}{*}{ Experiment } & \multicolumn{2}{|c|}{$\operatorname{ADC}(2)$} & \multicolumn{2}{|c|}{ CVS-ADC(2) } & \multirow[b]{2}{*}{$\Delta \mathrm{CVS}$} \\
\hline & & & Energy & $\Delta \operatorname{Expt}$ & Energy & $\Delta \mathrm{Expt}$ & \\
\hline $\mathrm{CH}_{4}$ & $1 s \rightarrow 3 p$ & $288.00^{66}$ & 290.57 & 2.67 & 290.63 & 2.74 & 0.07 \\
\hline \multirow[t]{2}{*}{$\mathrm{NH}_{3}$} & $1 s \rightarrow 3 a\left(a_{1}\right)$ & $400.66^{66}$ & 402.68 & 2.22 & 402.72 & 2.26 & 0.04 \\
\hline & $1 s \rightarrow 3 p(e)$ & $402.33^{66}$ & 404.38 & 2.25 & 404.41 & 2.28 & 0.03 \\
\hline \multirow[t]{2}{*}{$\mathrm{H}_{2} \mathrm{O}$} & $1 s \rightarrow 4 a_{1}$ & $534.00^{66}$ & 535.36 & 1.72 & 535.36 & 1.73 & 0.01 \\
\hline & $1 s \rightarrow 2 b_{2}$ & $535.90^{66}$ & 537.20 & 1.67 & 537.20 & 1.67 & 0.00 \\
\hline $\mathrm{HF}$ & $1 s \rightarrow \sigma *$ & $687.40^{67}$ & 687.86 & 1.07 & 687.84 & 1.04 & -0.02 \\
\hline $\mathrm{Ne}$ & $1 s \rightarrow 3 p$ & $867.12^{68}$ & 866.71 & 0.54 & 866.65 & 0.47 & -0.07 \\
\hline
\end{tabular}

TABLE II. Comparison of ionisation potentials obtained with $\mathrm{ADC}(2)$ and CVS-ADC(2). Energies are in eV thoughout. ${ }^{\mathrm{a}}$ For computational details see Table [

\begin{tabular}{lcccccc}
\hline & & \multicolumn{4}{c}{$\mathrm{ADC}(2)$} & \multicolumn{3}{c}{ CVS-ADC $(2)$} & \\
\cline { 4 - 6 } Molecule & Experiment & Energy & $\Delta$ Expt & Energy & $\Delta$ Expt & $\Delta$ CVS \\
\cline { 2 - 6 } $\mathrm{CH}_{4}$ & $290.76^{69}$ & 292.08 & 1.43 & 292.15 & 1.49 & 0.07 \\
$\mathrm{NH}_{3}$ & $405.52^{70}$ & 405.57 & 0.26 & 405.60 & 0.29 & 0.03 \\
$\mathrm{H}_{2} \mathrm{O}$ & $539.90^{66}$ & 538.34 & -1.19 & 538.33 & -1.20 & -0.01 \\
$\mathrm{HF}$ & $694.10^{71}$ & 691.36 & -2.13 & 691.36 & -2.17 & -0.04 \\
$\mathrm{Ne}$ & $870.09^{72}$ & 866.39 & -2.76 & 866.33 & -2.82 & -0.06 \\
\hline
\end{tabular}

experiment, and the difference in the CVS error between the two processes is smaller still.

\section{CONCLUSIONS AND OUTLOOK}

A simple Rayleigh-Quotient-based scheme for expanding eigenstates obtained with the core-valence separation (CVS) scheme to full space eigenstates for the $\operatorname{ADC}(n)$ hierarchy has been developed and implemented within the framework of the adcc python module ${ }^{39}$ This scheme is general and could be applied to relaxing the CVS approximation in other contexts such as coupled-cluster approaches and linear-response time-dependent densityfunctional theory. Using this approach, the error imposed by the CVS approximation has been investigated for $K$ edge absorption spectrum calculations, yielding discrepancies in energy of $-0.4 \mathrm{eV}$ to $0.7 \mathrm{eV}$, with exact value depending on the ADC level, basis set, and element in consideration.

The CVS error can to leading order be identified with two kinds of coupling terms, which are neglected in the CVS-ADC matrix, namely (1) cv-ov terms coupling single core- and single valence-excitations and (2) cv-cvcv terms, which describe the interaction of single and double core-excitations. Neglecting cv-ov and cv-cvcr has counteracting effects, since the cv-ov terms were identified to increase transition energies and the cv-cvcv to decrease them. Including cv-cvcv terms only, as is done in some CVS implementations, can thus yield a larger CVS error for the $1 s$-core transitions discussed in this article. Large cv-ov and cv-cvcv couplings were further identified to be a consequence of a balanced description of core and valence region. Suitable basis sets for a description of core-excitations at CVS level therefore give rise to large values of equal magnitude for these couplings, resulting in a small CVS error albeit a good description of the physics. This has been shown to be the case for basis sets with additional tight functions of a core-polarising nature.

The CVS error is typically larger for $\operatorname{ADC}(2)$ than for $\operatorname{ADC}(2)-x$ or $\operatorname{ADC}(3)$, and focusing on the carbon and oxygen edge of a set of representative compounds we report error spreads of maximally $0.02 \mathrm{eV}$ for bound excitations and ionisations, separately, with absolute errors being around $0.2 \mathrm{eV}$ for suitably core-polarised basis sets. Errors for the transition to extremely diffuse states are systematically about $0.02-0.03 \mathrm{eV}$ lower. Larger deviations from the typical CVS error on the carbon edge are only noted for $\mathrm{CO}$ and $\mathrm{CS}$. This has been attributed to the strong changes of electronic structure surrounding triple bounds. For most practical considerations the CVS approximation only yields a small error, which amounts to a mere a scalar shift of the full spectrum. The size of this shift is at least an order of magnitude smaller than the error with respect to experiment, making the 
CVS approximation a justified tool for the calculation of core-excitation spectra.

\section{ACKNOWLEDGMENTS}

The authors thank Andreas Dreuw and Jochen Schirmer for stimulating discussions. T.F. acknowledges financial support from the Swedish Research Council (Grant No. 2017-00356). This project has received funding from the European Research Council (ERC) under the European Union's Horizon 2020 research and innovation program (grant agreement No 810367).

\section{AIP PUBLISHING DATA SHARING POLICY}

The data that support the findings of this study are openly available on github (https://github.com/ mfherbst/cvs-relaxation-scripts) and have been deposited on Zenodo with DOI 10.5281/zenodo.3824506.

\section{APPENDIX: LEADING-ORDER PERTURBATIVE CORRECTION TO THE CVS EXCITATION ENERGIES}

The full ADC matrix can be written as $2 \times 2$ blocks

$$
\mathbf{M}=\left(\begin{array}{cc}
\mathbf{M}^{\text {core }} & \mathbf{C} \\
\mathbf{C}^{\dagger} & \mathbf{M}^{\text {rest }}
\end{array}\right),
$$

where $\mathbf{M}^{\text {core }}$ consists of the blocks kept in our CVS scheme, i.e. the ones involving only cv singly and cvov doubly excited configurations, $\mathbf{M}^{\text {rest }}$ collects all others (ov, cvcv, ovov) and $\mathbf{C}$ denotes the coupling between both classes. In this partitioning $\mathbf{M}^{\text {core }}=\mathbf{m}+\mathbf{D}$, where $\mathbf{m}$ is the CVS matrix as defined in Section II Notice that both $\mathbf{D}$ and $\mathbf{C}$ are zero if one neglects the coupling of core and valence orbitals via the Coulomb kerne ${ }^{37}$ and thus represents the terms missed by a CVS treatment. To apply perturbation theory we split $\mathbf{M}$ as

$$
\mathbf{M}=\underbrace{\left(\begin{array}{cc}
\mathbf{m} & 0 \\
0 & \mathbf{M}^{\text {rest }}
\end{array}\right)}_{=\mathbf{M}^{(0)}}+\underbrace{\left(\begin{array}{cc}
\mathbf{D} & \mathbf{C} \\
\mathbf{C}^{\dagger} & 0
\end{array}\right)}_{=\mathbf{M}^{(1)}} .
$$

As reference states we take the CVS vectors $\vec{x}_{i}$ from Equation (2), which are eigenstates of $\mathbf{M}^{(0)}$ with eigenvalue $\omega_{i}$ when extended by rows of zeros. In the following we restrict ourselves to the leading-order contribution in the perturbation $\mathbf{M}^{(1)}$, which amounts to keeping only the $\mathrm{ADC}(1)$ terms in $\mathbf{C}$ and neglecting $\mathbf{D}$ completely, where the lowest-order terms are at $\mathrm{ADC}(2)$ level. With this simplification the correction for the excitation energy of $\vec{x}_{i}$ is to second order

$$
\sum_{I} \frac{\left|\vec{x}_{i}^{\dagger} \mathbf{C} \vec{y}_{I}\right|^{2}}{\omega_{i}-E_{I}}
$$

In this expression $I$ runs over eigenstates $\vec{y}_{I}$ of $\mathbf{M}^{\text {rest }}$ with corresponding eigenvalues $E_{I}$. Since $E_{I}$ is to leading order given by orbital energy differences, $E_{I}<\omega_{i}$ for $I$ being an ov-dominated excitation and $E_{I}>\omega_{i}$ for $I$ being a cvcv-dominated excitation. For ovov-dominated excitations the coupling term in the numerator becomes zero at ADC(1) level, such that these terms do not contribute to lowest order. Overall including the neglected cv-ov coupling therefore pushes the energy of the coreexcitations up, the cv-cvcv coupling pushes the energy down and the cv-ovov coupling has little effect.

${ }^{1}$ U. Bergmann, V. K. Yachandra, J. Yano, and (Editors), X-Ray Free Electron Lasers: Applications in Materials, Chemistry, and Biology (The Royal Society of Chemistry, 2017).

${ }^{2}$ J. Stöhr, NEXAFS Spectroscopy (Springer, Berlin, 1992).

${ }^{3}$ J. A. van Bokhoven, C. Laberti, and (Editors), X-Ray Absorption and X-Ray Emission Spectroscopy: Theory and Applications (John Wiley \& Sons, 2016).

${ }^{4}$ P. M. Kraus, M. Zürch, S. K. Cushing, D. M. Neumark, and S. R. Leone, Nat. Rev. Chem. 2, 82 (2018).

${ }^{5}$ P. Norman and A. Dreuw, Chem. Rev. 118, 7208 (2018).

${ }^{6}$ G. Hähner, Chem. Soc. Rev. 35, 1244 (2006).

${ }^{7}$ M. Stener, G. Fronzoni, and M. de Simone, Chem. Phys. Lett. 373, 115 (2003).

${ }^{8}$ N. A. Besley and F. A. Asmuruf, Phys. Chem. Chem. Phys. 12, 12024 (2010).

${ }^{9}$ K. Lopata, E. van Kuiken, M. Khalil, and N. Govind, J. Chem. Theory Comput. 8, 3284 (2012).

${ }^{10}$ L. Triguero, L. G. M. Pettersson, and H. Ågren, J. Phys. Chem. A 102, 10599 (1998).

${ }^{11}$ I. Seidu, S. P. Neville, M. Kleinschmidt, A. Heil, C. M. Marian, and M. S. Schuurman, J. Chem. Phys. 151, 144104 (2019).

${ }^{12}$ R. Peng, A. V. Copan, and A. Y. Sokolov, J. Phys. Chem. A 123, 1840 (2019).

${ }^{13}$ S. Coriani, T. Fransson, O. Christiansen, and P. Norman, J. Chem. Theory Comput. 8, 1616 (2012).

${ }^{14}$ N. A. Besley, Chem. Phys. Lett. 542, 42 (2012).

${ }^{15}$ S. Coriani and H. Koch, J. Chem. Phys. 143, 181103 (2015).

${ }^{16}$ M. L. Vidal, X. Feng, E. Epifanovsky, A. I. Krylov, and S. Coriani, J. Chem. Theory Comput. 15, 3117 (2019).

${ }^{17}$ L. S. Cederbaum, W. Domcke, and J. Schirmer, Phys. Rev. A 22, 206 (1980).

${ }^{18}$ A. Barth and J. Schirmer, J. Phys. B: At. Mol. Phys. 18, 867 (1985).

${ }^{19}$ A. B. Trofimov, T. É. Moskovskaya, E. V. Gromov, N. M. Vitkovskaya, and J. Schirmer, J. Struct. Chem. 41, 483 (2000).

${ }^{20}$ J. Wenzel, M. Wormit, and A. Dreuw, J. Comp. Chem. 35, 1900 (2014).

${ }^{21}$ K. J. Oosterbaan, A. F. White, and M. Head-Gordon, J. Chem. Phys. 149, 044116 (2018).

${ }^{22}$ J. Wenzel, M. Wormit, and A. Dreuw, J. Chem. Theory Comput. 10, 4583 (2014).

${ }^{23}$ S. Banerjee and A. Sokolov, J. Chem. Phys. 151, 224112 (2019).

${ }^{24}$ J. Kauczor, P. Norman, O. Christiansen, and S. Coriani, J. Chem. Phys. 139, 211102 (2013).

${ }^{25}$ D. R. Rehn, A. Dreuw, and P. Norman, J. Chem. Theory Comput. 13, 5552 (2017).

${ }^{26}$ D. R. Nascimento and A. E. DePrince, J. Phys. Lett. 8, 2951 (2017).

${ }^{27}$ M. Kadek, L. Konecny, B. Gao, M. Repisky, and K. Ruud, PhysChemChemPhys 17, 22566 (2015).

${ }^{28}$ T. Fransson, D. Burdakova, and P. Norman, Phys. Chem. Chem. Phys. 18, 13591 (2016).

${ }^{29}$ Y. Saad, Numerical methods for large eigenvalue problems, 2nd ed. (SIAM Publishing, 2011).

${ }^{30}$ J. Schirmer and A. B. Trofimov, J. Chem. Phys. 120, 11449 (2004). 
${ }^{31}$ A. B. Trofimov, I. L. Krivdina, J. Weller, and J. Schirmer, Chem. Phys. 329, 1 (2006).

${ }^{32}$ J. Wenzel, A. Holzer, M. Wormit, and A. Dreuw, J. Chem. Phys. 142, 214104 (2015).

${ }^{33}$ J. Wenzel and A. Dreuw, J. Chem. Theory Comput. 12, 1314 (2016).

${ }^{34}$ S. P. Neville, V. Averbukh, M. Ruberti, R. Yun, S. Patchkovskii, M. Chergui, A. Stolow, and M. S. Schuurman, J. Chem. Phys. 145, 144307 (2016).

${ }^{35}$ T. Fransson and A. Dreuw, J. Chem. Theory Comput. 15, 546 (2019)

${ }^{36}$ A. Dreuw and M. Wormit, WIREs Comput. Mol. Sci. 5, 82 (2015)

${ }^{37} \mathrm{~J}$. Wenzel, Development and Implementation of Theoretical Methods for the Description of Electronically Core-Excited States, Ph.D. thesis, Universität Heidelberg (2016).

${ }^{38}$ W. J. Hehre, R. F. Stewart, and J. A. Pople, The Journal of Chemical Physics 51, 2657 (1969).

${ }^{39}$ M. F. Herbst, M. Scheurer, T. Fransson, D. R. Rehn, and A. Dreuw, "adcc: A versatile toolkit for rapid development of algebraic-diagrammatic construction methods," (2020).

${ }^{40}$ C. Møller and M. S. Plesset, Phys. Rev. 46, 618 (1934).

${ }^{41}$ T. H. Dunning, J. Chem. Phys. 90, 1007 (1989).

${ }^{42}$ Y. Shao, Z. Gan, E. Epifanovsky, A. T. B. Gilbert, M. Wormit, J. Kussmann, A. W. Lange, A. Behn, J. Deng, X. Feng, D. Ghosh, M. Goldey, P. R. Horn, L. D. Jacobson, I. Kaliman, R. Z. Khaliullin, T. Kús, A. Landau, J. Liu, E. I. Proynov, Y. M. Rhee, R. M. Richard, M. A. Rohrdanz, R. P. Steele, E. J. Sundstrom, H. L. Woodcock III, P. M. Zimmerman, D. Zuev, B. Albrecht, E. Alguire, B. Austin, G. J. O. Beran, Y. A. Bernard, E. Berquist, K. Brandhorst, K. B. Bravaya, S. T. Brown, D. Casanova, C.-M. Chang, Y. Chen, S. H. Chien, K. D. Closser, D. L. Crittenden, M. Diedenhofen, R. A. DiStasio Jr., H. Dop, A. D. Dutoi, R. G. Edgar, S. Fatehi, L. Fusti-Molnar, A. Ghysels, A. Golubeva-Zadorozhnaya, J. Gomes, M. W. D. HansonHeine, P. H. P. Harbach, A. W. Hauser, E. G. Hohenstein, Z. C. Holden, T.-C. Jagau, H. Ji, B. Kaduk, K. Khistyaev, J. Kim, J. Kim, R. A. King, P. Klunzinger, D. Kosenkov, T. Kowalczyk, C. M. Krauter, K. U. Lao, A. Laurent, K. V. Lawler, S. V. Levchenko, C. Y. Lin, F. Liu, E. Livshits, R. C. Lochan, A. Luenser, P. Manohar, S. F. Manzer, S.-P. Mao, N. Mardirossian, A. V. Marenich, S. A. Maurer, N. J. Mayhall, C. M. Oana, R. Olivares-Amaya, D. P. O'Neill, J. A. Parkhill, T. M. Perrine, R. Peverati, P. A. Pieniazek, A. Prociuk, D. R. Rehn, E. Rosta, N. J. Russ, N. Sergueev, S. M. Sharada, S. Sharmaa, D. W. Small, A. Sodt, T. Stein, D. Stück, Y.-C. Su, A. J. W. Thom, T. Tsuchimochi, L. Vogt, O. Vydrov, T. Wang, M. A. Watson, J. Wenzel, A. White, C. F. Williams, V. Vanovschi, S. Yeganeh, S. R. Yost, Z.-Q. You, I. Y. Zhang, X. Zhang, Y. Zhou, B. R. Brooks, G. K. L. Chan, D. M. Chipman, C. J. Cramer, W. A. Goddard III, M. S. Gordon, W. J. Hehre, A. Klamt, H. F. Schaefer III, M. W. Schmidt, C. D. Sherrill, D. G. Truhlar, A. Warshel, X. Xua, A. Aspuru-Guzik, R. Baer, A. T. Bell, N. A. Besley, J.-D. Chai, A. Dreuw, B. D. Dunietz, T. R. Furlani, S. R. Gwaltney, C.-P. Hsu, Y. Jung, J. Kong, D. S. Lambrecht, W. Liang, C. Ochsenfeld, V. A. Rassolov, L. V. Slipchenko, J. E. Subotnik, T. Van Voorhis, J. M. Herbert, A. I. Krylov, P. M. W. Gill, and M. Head-Gordon, Mol. Phys. 113, 184 (2015).

${ }^{43}$ M. F. Herbst and M. Scheurer, "adc-connect/adcc v0.13.2," (2019).

${ }^{44}$ Q. Sun, T. C. Berkelbach, N. S. Blunt, G. H. Booth, S. Guo, Z. Li, J. Liu, J. D. McClain, E. R. Sayfutyarova, S. Sharma, S. Wouters, and G. K.-L. Chan, WIREs Comput. Mol. Sci. 8, 1340 (2018).

${ }^{45} \mathrm{M}$. F. Herbst and T. Fransson, "Implementation and examples illustrating Rayleigh-Quotient-based CVS relaxation," https:// github.com/mfherbst/cvs-relaxation-scripts
${ }^{46}$ R. Krishnan, J. S. Binkley, R. Seeger, and J. A. Pople, J. Chem. Phys. 72, 650 (1980).

${ }^{47}$ D. E. Woon and T. H. Dunning, J. Chem. Phys. 103, 4572 (1995).

${ }^{48}$ R. A. Kendall, T. H. Dunning, and R. J. Harrison, J. Chem. Phys. 96, 6796 (1992).

${ }^{49}$ M. Douglas and N. Kroll, Ann. Phys. 82, 89 (1974).

${ }^{50}$ B. A. Hess, Phys. Rev. A 33, 3742 (1986).

${ }^{51}$ G. Jansen and B. A. Hess, Phys. Rev. A 39, 6016 (1989).

52 "Dalton, a molecular electronic structure program," Release DALTON2016.alpha (2015), see http://daltonprogram.org.

${ }^{53}$ K. Aidas, C. Angeli, K. L. Bak, V. Bakken, R. Bast, L. Boman, O. Christiansen, R. Cimiraglia, S. Coriani, P. Dahle, E. K. Dalskov, U. Ekström, T. Enevoldsen, J. J. Eriksen, P. Ettenhuber, B. Fernández, L. Ferrighi, H. Fliegl, L. Frediani, K. Hald, A. Halkier, C. Hättig, H. Heiberg, T. Helgaker, A. C. Hennum, H. Hettema, E. Hjertenæs, S. Høst, I.-M. Høyvik, B. Iozzi, M. F. Jansik, H. J. Aa. Jensen, D. Jonsson, P. Jørgensen, J. Kauczor, S. Kirpekar, T. Kjærgaard, W. Klopper, S. Knecht, R. Kobayashi, H. Koch, J. Kongsted, A. Krapp, K. Kristensen, A. Ligabue, O. B. Lutnæs, J. I. Melo, K. V. Mikkelsen, R. H. Myhre, C. Neiss, C. B. Nielsen, P. Norman, J. Olsen, J. M. H. Olsen, A. Osted, M. J. Packer, F. Pawlowski, T. B. Pedersen, P. F. Provasi, S. Reine, Z. Rinkevicius, T. A. Ruden, K. Ruud, V. Rybkin, P. Salek, C. C. M. Samson, A. Sánchez de Merás, T. Saue, S. P. A. Sauer, B. Schimmelpfennig, K. Sneskov, A. H. Steindal, K. H. Sylvester-Hvid, P. R. Taylor, A. M. Teale, E. I. Tellgren, D. P. Tew, A. J. Thorvaldsen, L. Thøgersen, O. Vahtras, M. A. Watson, D. J. D. Wilson, M. Ziolkowski, and H. Ågren, WIREs Comput. Mol. Sci. 4, 269 (2014).

${ }^{54}$ B. Peng, P. J. Lestrange, J. J. Goings, M. Craicato, and X. S. Li, J. Chem. Theory Comput. 11, 4146 (2015).

${ }^{55}$ M. Schindler and W. Kutzelnigg, J. Chem. Phys. 76, 1919 (1982).

${ }_{56}$ A. E. A. Fouda and N. A. Besley, Theor. Chem. Acc. 137, 6 (2018).

${ }^{57}$ M. A. Ambroise and F. Jensen, J. Chem. Theory Comput. (2018).

${ }^{58}$ T. Fransson, S. Coriani, O. Christiansen, and P. Norman, J. Chem. Phys. 138, 124311 (2013).

${ }^{59}$ J. G. Hill, International Journal of Quantum Chemistry 113, 21 (2013).

${ }^{60}$ J. D. Wadey and N. A. Besley, J. Chem. Theory Comput. 10, 4557 (2014).

${ }^{61}$ R. Sarangi, M. L. Vidal, S. Corniani, and A. I. Krylov, (2020).

${ }^{62}$ Files defining the modified basis sets are available from Reference 45

${ }^{63} \mathrm{~F}$. Jensen, Wiley Interdisciplinary Reviews: Computational Molecular Science 3, 273 (2013).

${ }^{64}$ J. F. Stanton and J. Gauss, J. Chem. Phys. 111, 8785 (1999).

${ }^{65}$ S. Coriani, O. Christiansen, T. Fransson, and P. Norman, Phys. Rev. A 85 (2012).

${ }^{66}$ J. Schirmer, A. B. Trofimov, K. J. Randall, J. Feldhaus, A. M. Bradshaw, Y. Ma, C. T. Chen, and F. Sette, Phys. Rev. A 47, 1136 (1993)

${ }^{67}$ A. P. Hitchcock and C. E. Brion, J. Phys. B: At. Mol. Phys. 14, 4399 (1981)

${ }^{68}$ M. Coreno, R. Avaldi, R. Camilloni, K. C. Prince, M. de Simone, J. Karvonen, R. Rolle, and S. Simonucci, Phys. Rev. A 59, 2494 (1999).

${ }^{69}$ M. Tronc, G. C. King, and F. H. Read, J. Phys. B: At. Mol. Phys. 12, 137 (1979).

${ }^{70}$ B. E. Mills, R. L. Martin, and D. A. Shirley, J. Am. Chem. Soc. 98, 2380 (1976).

${ }^{71}$ A. A. Bakke, H.-W. Chen, and W. L. Jolly, J. Elec. Spec. Rel. Phenom. 20, 333 (1980).

${ }^{72}$ A. P. Hitchcock and C. E. Brion, J. Phys. B: At. Mol. Phys. 13, 3269 (1980).

${ }^{73}$ A. Thiel, J. Schirmer, and H. Köppel, J. Chem. Phys. 119, 2088 (2003). 